Usage, Usability, and Utility of 3D City Models, 02008 (2012)

DOI: $10.1051 / 3 \mathrm{u} 3 \mathrm{~d} / 201202008$

(C) Owned by the authors, published by EDP Sciences, 2012

\title{
EnVIE project: A framework to produce urban data at large scale
}

\author{
V. Tourre ${ }^{1}$, P. Cadio ${ }^{2}$, G. Caniot ${ }^{3}$ and C. Papin ${ }^{4}$ \\ 1 LUNAM Université, École Centrale de Nantes, CERMA UMR CNRS 1563, 1, rue de la Noë, \\ BP. 92101, 44321 Nantes Cedex 3, France \\ 2 MGDIS, Parc d'Innovation Bretagne Sud, CP 10, 56038 Vannes, France \\ 3 MeteoDyn, 14 Bd. Winston Churchill, 44100 Nantes, France \\ 4 SIRADEL, 3 allée Adolphe Bobierre 35043 Rennes, France
}

\begin{abstract}
The decision making process related to urban areas synthetizes a lot of data taking into account numerous parameters. The indicators produced from simulations or data mining on 3D city models (3DCM) can be a great help into these decision-making processes. The problem is to acquire and process the 3DCM in order to produce these indicators at city scale. The EnVIE project proposes a framework to perform the acquisition of 3DCM and then to produce 3D or 2D metadata on these models. As several city councils have been interviewed in the scope of the project to describe their real needs, the computed metadata are directly usable into the decision-making process. The main contribution of this framework is to merge existing techniques to build a pipeline allowing to compute lighting and wind simulation, and to extract socioeconomic metadata with data-mining techniques. This on-going work builds a link between indicators at the building scale and 2D indicators at larger scale from GIS, and therefore is a step towards an indicator production process at full-scale range.
\end{abstract}

\section{INTRODUCTION}

Thinking, refurbishing or building the city of tomorrow remains a tricky issue. More and more people live in urban areas worldwide and want an improved quality of life: more work, better facilities, etc. In parallel, most of the cities see their environmental, socioeconomic and socio-demographic indicators negatively impacted since they are affected by too many rapid changes: pollutions, waste and energy consumption, traffics, type and geographical distribution of population...

In that context, the $3 \mathrm{D}$ modeling of complex urban communities is a well-known and powerful tool for decision-making and communication. It allows displaying and interacting with city features at different scales (buildings, trees, road network, street furniture...), simulating some scenarios (urban planning, deployment or optimization of telecom, energy or transport infrastructures, risks management...) while making participating citizens to decision-making or just raising public awareness of public programs and expenses.

However, it still lacks a way to seamlessly product, integrate and merge into such a 3D representation some heterogeneous data that would bring more value to the 3D model. Such complementary attributes are for instance outcomes from simulation tools that use 3D data as inputs (wind resource mapping and forecasting, radio propagation models, noise pollution, street lighting, etc.), but also from results of detection or classification algorithms based on processing of aerial, satellite or terrestrial High Resolution (HR) imagery and LIDAR. 3D city models (3DCM) can also be enriched with higher level (semantic) descriptors of the environment or with some understandable eco-labels obtained through cross-data analysis: e.g. the global heat losses in 3D,

This is an Open Access article distributed under the terms of the Creative Commons Attribution License 2.0, which permits unrestricted use, distribution, and reproduction in any medium, provided the original work is properly cited. 


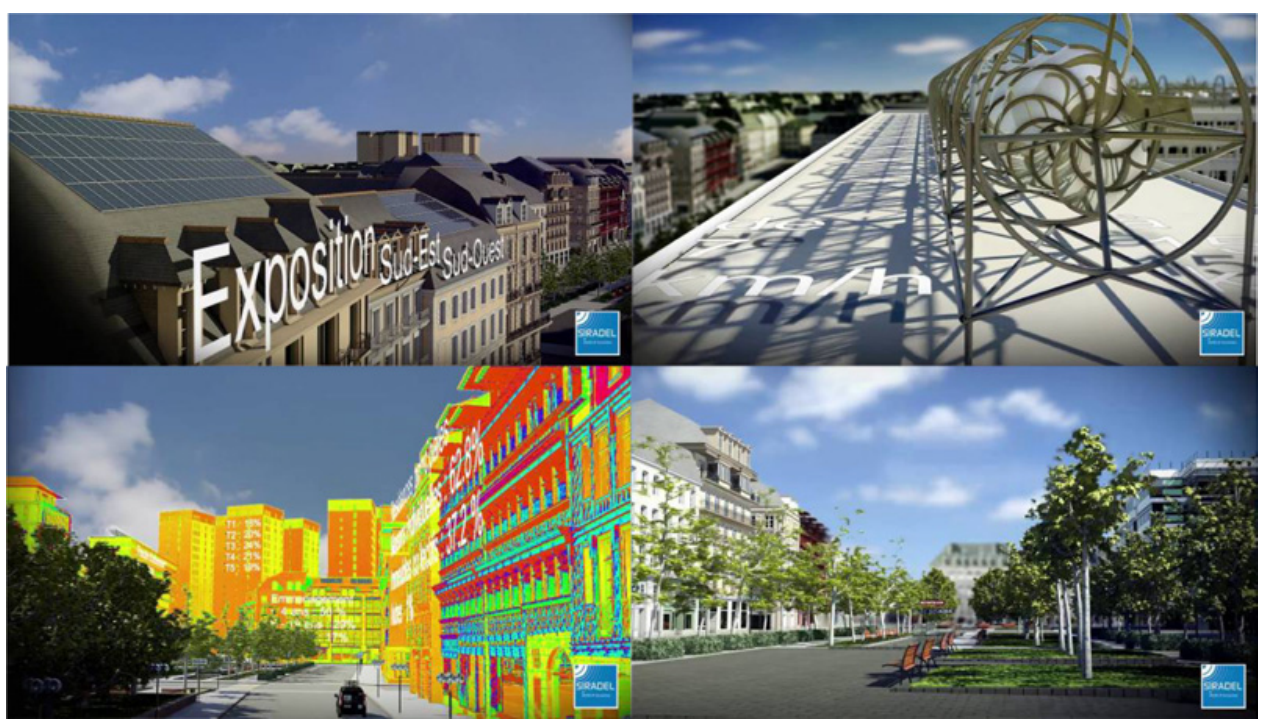

Figure 1. Illustrations of some topics addressed as part of the EnVIE project: at top, potential energy (sun exposure, wind), at bottom, heat losses combined with socioeconomic data and environmental quality indicators.

i.e. a unique tag encompassing both losses at the roof and façades levels (aerial and terrestrial thermal imaging), but also taking into account the building superstructures so as to refine the analysis. Another class of high level descriptors attempts to qualify and rank the human perception and acceptability of his surrounding environment (comfort or stress). It is based on the combined use of features from 3D geometry (visibility, pavement width, building height...) and additional inputs like the presence of trees, the wind and temperature fields, lighting, the noise pollution or others.

The next step consists in combining those outcomes with some socioeconomic and demographic attributes (land registry, census data or from geo-marketing data mining...). This ultimate goal is straight addressed by the EnVIE project ("Enrichment of digital 3D cities with environmental eco-indicators"). This 2-years long project started in 2011 and addresses the four following topics:

- 3D modeling of urban areas.

- Potential energy (contributions and losses).

- Environmental quality (human perception of comfort).

- Economic and social situation.

All these individual outcomes are merged and finally integrated into the 3D model to offer much more possibilities to analyze and understand the city. This will result in an enriched 3DCM allowing multiscale and multi-criteria representation and analysis for decision-making and communication purposes.

A working group that encompasses different urban communities and cities (Rennes Métropole, Nantes Métropole, Sant-Grégoire and Cesson-Sévigné) has been established at the project start. It allowed the EnVIE Consortium to gather a wide range of expressed needs from various technical services (GIS, Environment...) and elected representatives.

This paper describes in section 2 the state of the art before introducing the overall EnVIE framework in section 3. The first results are illustrated in section 4 and discussed in section 5. Finally, section 6 concludes. 


\section{PREVIOUS WORKS}

\subsection{D model acquisition}

Many markets ask for more immersivity and 3D city models gains always more traction. 3D models can also be used as a powerful interface to aggregate and display multiples levels of information. This requires an accurate building modeling, texture mapping (aerial or terrestrial images or results from scientific computations), insertion of various additional 3D objects, but also managing data complexity, structure and source-channel coding for enabling real-time navigation whatever the network and the rendering device is. In particular, more and more stakeholders ask for navigation at the street level and not only a simple bird's eyes view (communication, virtual tourism, Location-Based B2B or B2C Services...) associated with the need of both data fidelity and a strong feeling of realism.

$3 \mathrm{D}$ city models can be obtained by means of photogrammetry from aerial or satellite stereo images. $3 \mathrm{D}$ vectors or $3 \mathrm{D}$ coordinates of key points characterizing roofs, terrain or some infrastructures like roads are determined. For more realism, the Digital Terrain Model (DTM) as well as the roofs and the building's façades can be mapped with image textures. When pedestrian navigation is targeted, aerial imagery becomes not enough to achieve realistic façade texturing. A new type of acquisition systems is gaining traction: the Mobile Mapping Systems (MMS). MMS has been evolving quickly and can embed highly accurate multi-sensor platforms, equipped for instance with GPS/IMU/DMI localization systems, high-resolution RGB cameras, terrestrial LIDAR... As an example, the production chain of the SIRADEL's MMS [1] includes 3 successive stages: 3D model registration using laser data, occlusion detection using both laser and imagery sensors, and finally image merging. Figure 2 shows the MMS system once deployed over the vehicle roof.

The simplest class of 3D building models consists of grey walls and flat roofs extruded from 2D building outlines. They are quite simple to handle at a wide scale and are well-suited for GIS-oriented applications or simulation purposes (e.g. Computational Fluid Dynamics or CFD, radio propagation...).

More realism can be achieved both in geometry (roof superstructures, recesses in building façades, balconies...), and through texture mapping. Figure 2 shows that roofs can be modeled very accurately from high resolution aerial pictures ( $5 \mathrm{~cm}$ in the example at middle). This is of importance for instance when evaluating the potential energy of solar panel. The second step consisted in projecting aerial orthoimagery onto the roof. The use of aerial imagery is still relevant for façade texturing since resolution is getting better (ground point size less than $10 \mathrm{~cm}$ is now quite common, cf. Figure 2 below from $5 \mathrm{~cm}$ images). Generic (geo-typical) textures or aerial oblique (or not if overlapping between images is high) imagery can be used. But even whether the image resolution has getting better over the years, only using aerial images to texture façades appears to be not enough and perspective discrepancies at the street level are visually unpleasant. A terrestrial image-based approach has to be preferred, especially for street view navigation or monitoring.

As regards geometry, some past studies proposed algorithms for automatic computation of generic roofs based on Digital Elevation Model (DEM). More recently, façade segmentation and window detection techniques based on oblique aerial images have been proposed to improve the façade rendering, like in [2]. However, due to perspective and image resolution, this does not appear relevant for ensuring an accurate detection and extraction of objects that are not located in the plane of the façade, like balcony. Again, a terrestrial approach should be privileged.

\subsection{Large scale simulation}

Authors in [3] proposed a method for planning and optimization of new wideband radio networks over large geographical areas with deterministic tools. They presented a fast and robust propagation model that computes 3-D ray contributions using 3-D urban model. Experiments were conducted on an area of $64 \mathrm{~km}^{2}$. 

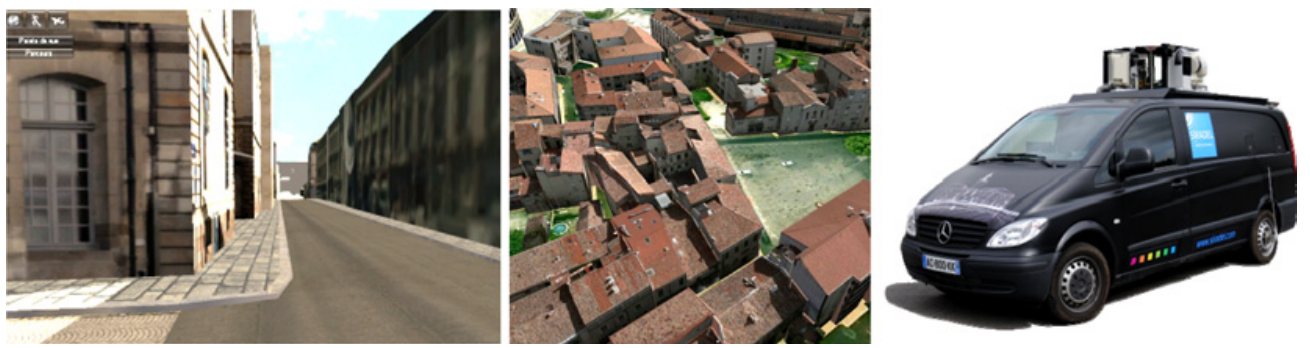

Figure 2. Limitations when texturing façades from aerial imagery. On the left: comparison between a façade textured with terrestrial images (see section 2.1) at the left side and aerial pictures $-15 \mathrm{~cm}$ - on the right (city of Rennes). At middle: accurate roof modeling and texturing from $5 \mathrm{~cm}$ images (data is property of the city of Nîmes, France). At right: SIRADEL's MMS.

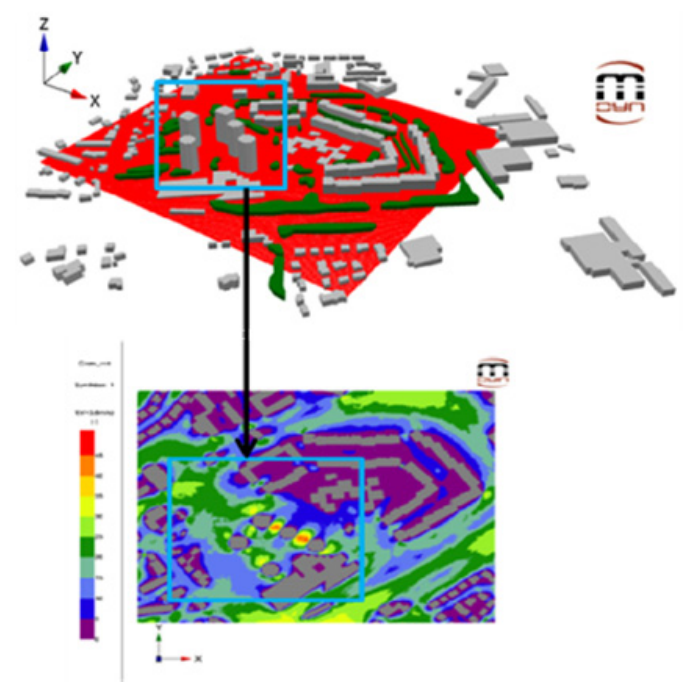

Figure 3. Wind Comfort map before urban renewal.

Meteodyn [4] has a broad experience in wind and solar simulation over a large domain. For example, wind comfort and insulation frequency was computed in Saint-Brieuc district:

\subsection{Indicator production}

In order to aid the decision making process, the results of the preceding simulations have to be translated into understandable indicators for the urban community managers. The ADEQUA [5] project have proposed a methodology to aid the decision making process during the building or the renewing of a residential area. The goal is to clearly present various indicators in order to show the complexity and the underlying mechanisms of the sustainable development. Following the same sustainable approach, the PACIBA project [6] presents a methodology and software in order to handle the early stages of conceptual design. Several physical phenomena are taken into account to preview what could be the energetic qualities of a small city area. 
European COST Action TU0801

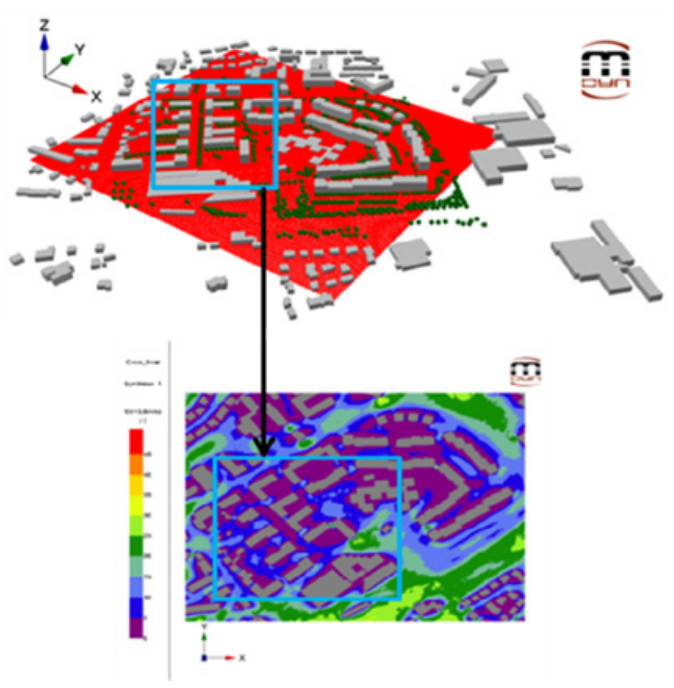

Figure 4. Wind Comfort map after urban renewal.

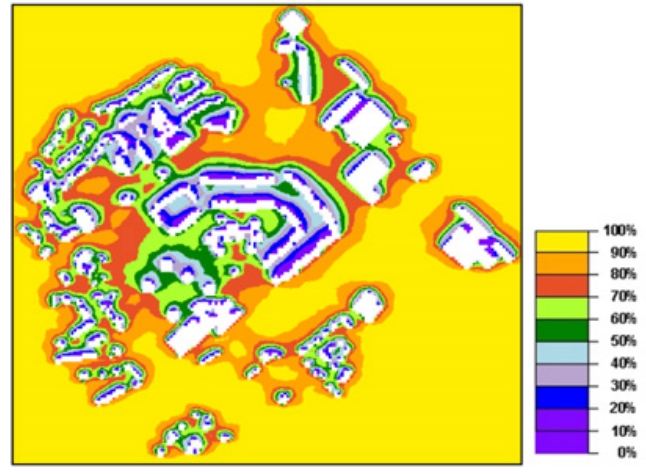

Figure 5. Annual Insulation Frequency before urban renewal.

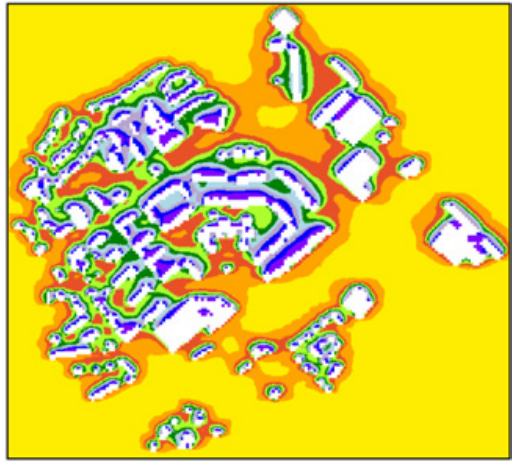

Figure 6. Annual Insulation Frequency after urban renewal. 


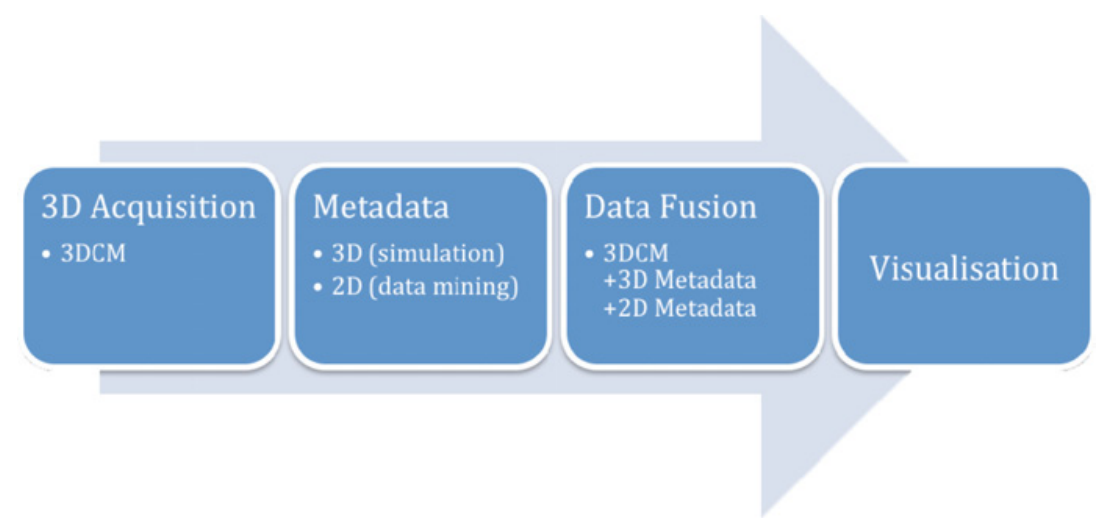

Figure 7. Overall EnVIE workflow.

\section{FRAMEWORK}

\subsection{D city modeling}

The 3D city model is the common base on which all EnVIE metadata has been aggregated. For partners, 3DCM is used in different ways: extracting descriptors of the city environment, input data for simulation or 3D container allowing displaying multiple data. For end-users, when combined with real-time 3D navigation capabilities, it enables benefiting from a smart user interface for interactive access to ecolabels. The 3D city model encompasses the terrain, the buildings (in LOD1), the vegetation, the street furniture and some administrative borders.

SIRADEL produced distinct city models from two representative areas, located respectively in Rennes and in Saint-Grégoire. These 2 samples are large enough to validate different use cases and are complementary in terms of contents (residential area, houses, tower blocks...)

As an example, the Figure below illustrates different levels of city modeling.

\subsection{Data exchange}

The interoperability problem is to exchange geometric data, to add metadata related to this geometric data, and then to exchange this metadata. So on one side, we have to send 2D or 3D geometric data to perform simulations and analysis, and on the other side, we have to keep semantic attributes in order to build a hierarchic structure of the city model. We have chosen to have two distinct data processes depending on the metadata dimensions, and two distinct files.

\subsection{D metadata - simulation}

The STL format is highly recognized for several simulators and has been chosen as the simulation format. The CityGML format adds a semantic level and hierarchic structure needed for using the metadata. We add a linking file to explicitly describe the relationship between STL triangle patches and CityGML entities; in order to retrieve the CityGML entities triangle patches belongs to. This linking file is a kind of "CityGML light" format containing a simple 3D geometric hierarchic structure, each entity embedding the set of STL triangle patch identifier and the CityGML entity identifier.

Therefore the STL file can be used to perform simulations onto the 3DCM and produce metadata over each triangle patches. Then, thanks to the linking file, the computed metadata can be associated to the corresponding CityGML entity. 


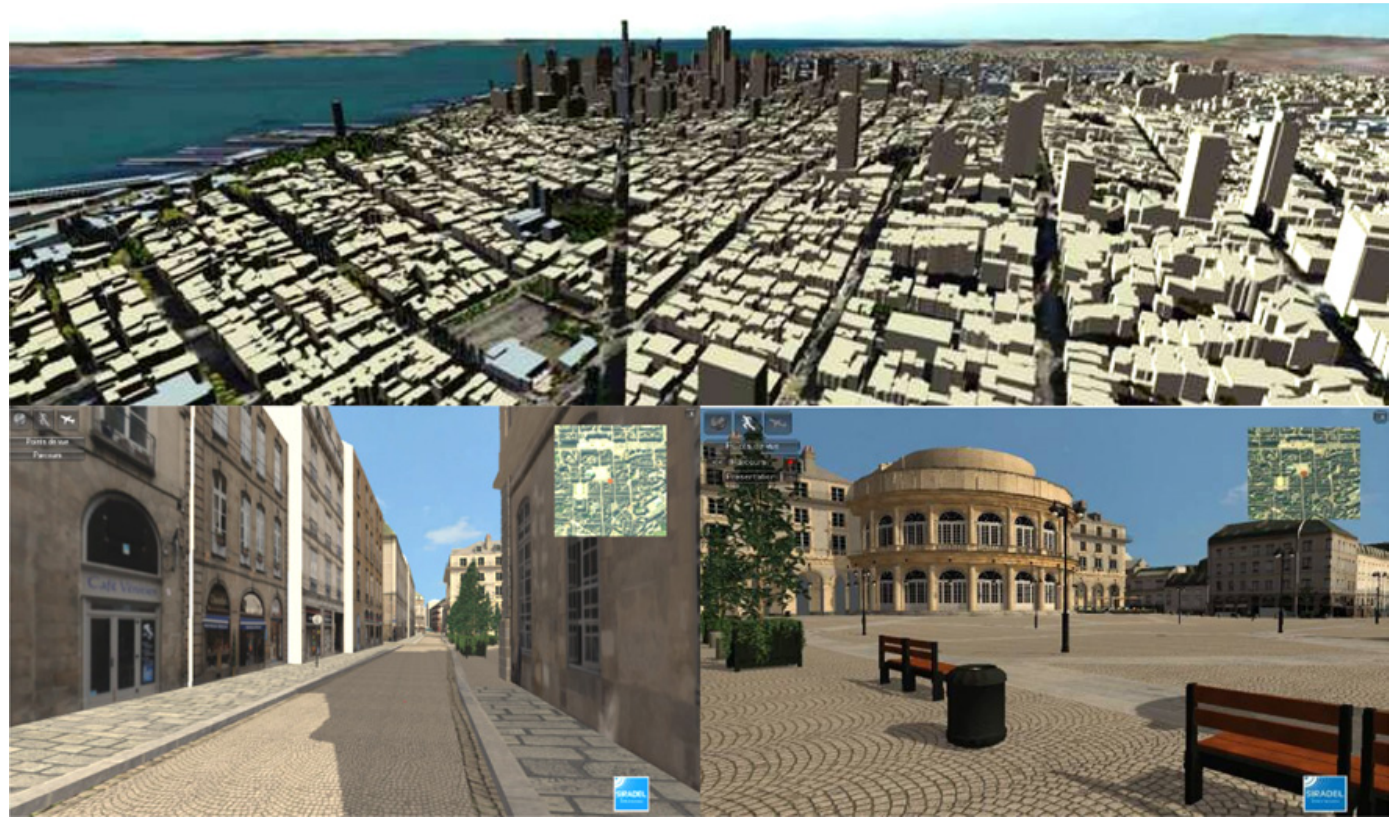

Figure 8. Different level of city modeling. At top, San Francisco, one of the thousands of cities in LOD $1^{1}$ produced by SIRADEL all around the world. At bottom-left, realistic 3D model of Rennes (France) in LOD2 and LOD3 with terrestrial texture acquired by a Mobile Mapping System (MMS) from SIRADEL. At bottom-right, 3D landmark and enrichment with street furniture.

\subsubsection{Metadata related to lighting and energy}

Wind characteristics and solar energy are computed using simulation software called Urbawind. Thanks to its powerful CFD (Computational Fluid Dynamics) solver, wind characteristics such as wind speed, wind direction and wind energy can be assessed over a large domain such as a district.

In order to compute direct solar energy for a very large domain, an optimized structure of the geometric objects (thanks to Bounding Volume Hierarchy) combined with fast computation on GPU (Graphical Processor Unit) gives us a shadow map for a sun position. By summing over a period, a frequency of shadow (or light) can be assessed. Solar energy from direct lighting can be computed from summing all beans taking into account energy a beam at each time step.

From the CAD file in STL format, wind and solar results are computed over roofs, façades and streets. Thanks to "CityGML light" format, as described below, the energy and lighting metadata related to CityGML entity can be sorted it out.

\subsubsection{Metadata related to pedestrian perception}

The STL data is converted into the specific data format needed by Solene. Solene is a lighting and thermic simulator based on radiosity, and able to handle visibility and comfort problems [7]. In the EnVIE project, the Solene simulator is used to compute indirect lighting used in lighting and energy metadata, but also the pedestrian visibility of specific buildings or vegetation, and the pedestrian thermic comfort.

\footnotetext{
${ }^{1}$ LOD: Level of Detail.
} 
The challenge of these metadata based on the pedestrian perception is describing a local perception represented in a 3D scene. A local perception is situated in space; this is the easy part to represent on a map or in a 3D scene, but also in time. The pedestrian perception depends on what the pedestrian has done before, and sometimes, on what he will do later or what he has planed to do. As the time dimension is difficult to take into account without any walkway or path clues, in this paper we focus on the visibility of specific building, because this indicator is less biased without the time dimension.

\subsection{D metadata - City inhabitants}

The aim of this project is to provide socio-demographic data within spatial environmental data. MGDIS applies their expertise in the value-addition on public data. Starting from public information made available by institutions (INSEE, Government-owned agencies, etc.), MGDIS is able to compute populations from areas, and to localize the profiles of people in their residence.

The advantage of this approach is to provide a detailed knowledge of population and housing currently present in the territory, in order to facilitate interpretation and diagnostic. This also enables an organization to identify possible actions toward population in the concerned living areas. For example, a tenant is not supposed to be contacted by a city for additional ecological equipment.

\subsubsection{Buildings identification from the $3 D$ representation}

The development of a parcel layer (data from the cadastre) is necessary to precisely locate 3D buildings in the city. This parcel identification allows the linking of a 3D representation of the building with cadastral information listed by the municipality. From this information, a profile of inhabiting is determined and thus allows identifying a population potentially present in the living area.

The algorithm consists in matching the map of parcels (land register), used to identify housing, with the actual map of the buildings, taking into account the constraints linked to the $3 \mathrm{D}$ representation of the city (joint ownership of buildings, etc.).

\subsubsection{The granularity of information}

Socio-demographic data can then be piled on this new 3D base map, which respects the city morphology and its cadastral representation.

To this date, providers of public data in France, such as INSEE, must respect the constraints of privacy edited by the national numeric privacy agency (CNIL), and publish aggregate data on the level of a territory. The finest geographical level published by INSEE is the IRIS, an infra-municipal territory representing a population of roughly 2,000 inhabitants, for municipalities with more than 10000 inhabitants. For municipalities with fewer than 10000 inhabitants, the level is the basic geographic Township.

In order to refine the result and correctly affect the inhabitants in the $3 \mathrm{D}$ city, information must be analyzed some more to obtain a given granularity. This granularity of information is obtained from algorithms developed by MGDIS, allowing the correspondence between the cadastral information at the address, and demographic information in IRIS. In the end, this allows the identification of populations present on that territory, together with the type of housing, and indicates to the user the level of robustness in the results.

\section{FIRST RESULTS}

This section illustrates some preliminary outcomes. Data integration in the final system is not taken into account at this rate. 

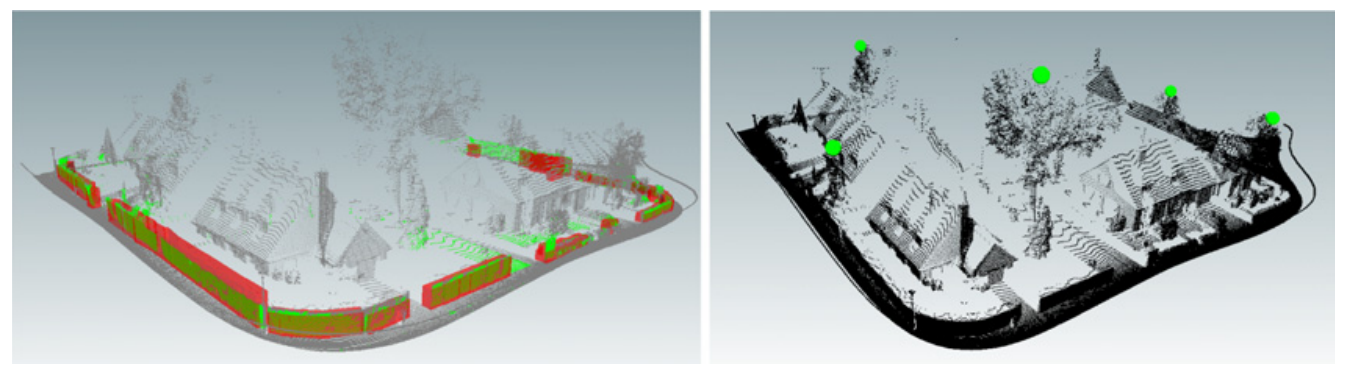

Figure 9. (left) Hedge extraction in a laser point cloud. Low vegetation points are represented in green and final hedges are in red, (right) Tree extraction in a laser point cloud. Green shows the top of the detected trees.

\subsection{Vegetation detection}

Detection and classification of the vegetation are commonly achieved using aerial imagery: e.g. nearinfrared imagery for detection and DEM for classification (high vegetation or low vegetation). For some objects, a terrestrial-based approach can be relevant as well. For example, trunks of trees are not visible from aerial views. In general, the tree location is assigned to the middle of the canopy and not to the middle of the trunk.

SIRADEL implemented a tree and hedges detection method from input data acquired on ground. Regarding hedge, "green areas" are first detected from terrestrial images. Then, hedges are geometrically extracted from the terrestrial laser points. The main issue in this step is to select only vegetation pixels from winter images, where branch and shadow colors are quite similar. The second stage consists in analyzing the geometrical characteristics of each individual hedge. Results are shown in Figure 9 (left).

The most common way to detect trees in terrestrial laser data is to extract their trunks by finding cylinders in the laser points $[8,9]$. However, trunks are often hidden behind hedges in residential areas. Our method includes a first filtering of potential tree points by analyzing a point projection image onto the ground. Results are shown in Figure 9 (right).

A terrestrial approach, when considered alone, is not enough but can complement well another processing. Aerial detection can be considered as a global method, while terrestrial detection helps improving accuracy in terms of position and dimension, at least when the object is visible from the street.

\subsection{Thermal imagery}

The MMS can provide a lot of information about the environment, especially thanks to additional sensors. This represents a unique capability to collect a wide amount of data from the ground. This allows adding knowledge into the 3D database. We focus here on thermal imagery. As well, these inputs will be combined with aerial imagery or simulation tools in order to derive eco-indicators (like insulation fault) that are embedded into the 3D city model.

\subsection{Lighting and Wind data}

The computed insulation frequency is displayed here using Red color as the maximum and Purple color as the minimum of insulation frequency for Saint-Grégoire neighborhood (Figures 10 and 11). Wind characteristics are also accessible here in a 2D plan. Results are shown figures 12 and 13. 


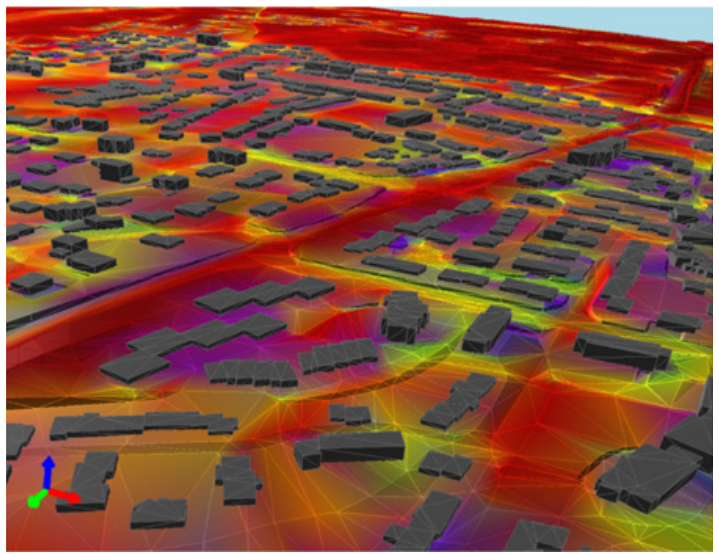

Figure 10. Daily Insulation frequency on the streets of Saint-Grégoire.

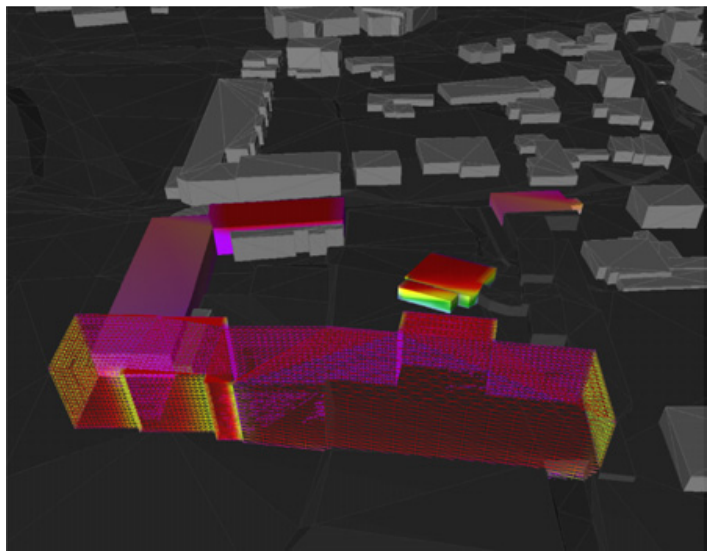

Figure 11. Daily Insulation frequency on building's facades of Saint-Grégoire.

\subsection{Interviews}

A working group that encompasses different urban communities and cities (Rennes Métropole, Nantes Métropole, Saint-Grégoire and Cesson-Sévigné) has been established at the beginning of the project. It allowed us to gather a wide range of expressed needs from various technical services (SIG, Environment...) and elected representatives.

A questionnaire has been sent to these end-users. It included three distinct parts: the first part provided a set of eco-indicators that could be derived and explored as part of EnVIE, with the possibility to rank each of them according to 5 levels. The second part proposed some combinations of ecoindicators given specific uses. A ranking of such different use cases and combinations was also asked for. The last part of the questionnaire was an open section in order to freely express any kind of comments.

As regards to the eco-indicators themselves, heat losses, the distance to services and urban climate comfort have been aroused the most interest. Some particular use cases were also preferred by the endusers. For instance, let us mention the analysis of the daily commute between home and work, local energy policy or the qualification of existing or new districts. All end-users favor smart but simple tools, easy-to-use and business-oriented. 


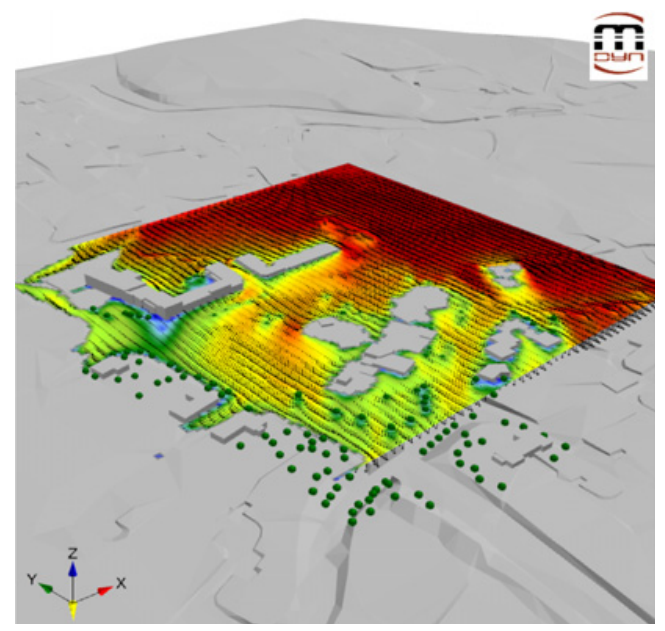

Figure 12. Directional Wind velocity on 2D Horizontal Plan on the streets of Saint-Grégoire.

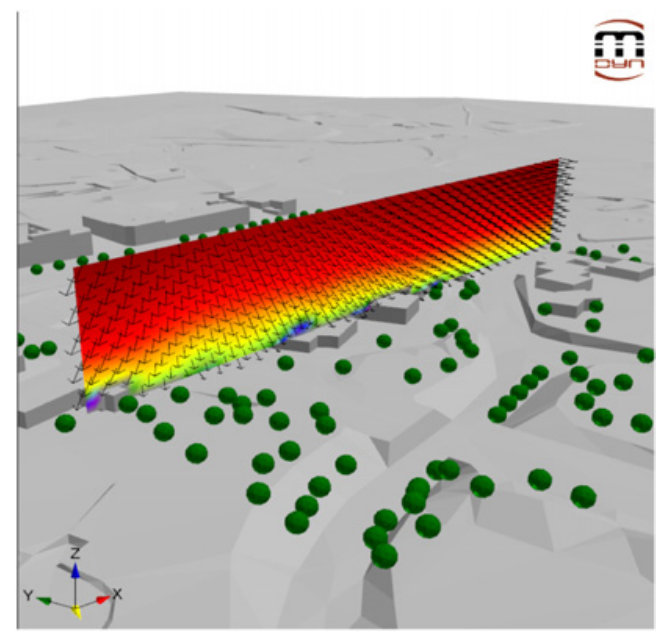

Figure 13. Directional Wind velocity on 2D Vertical Plan on the streets of Saint-Grégoire.

\subsection{First 2D metadata computations}

MGDIS currently works with communities by providing all the information needed for analysis and diagnosis of territories, starting from the data of institutions (INSEE, local authorities...). This data is structured and given added value by MGDIS, making them usable and understandable for elected members working in the definition of public policies.

Data extractions from this heap of public data identify the description of housing and population, by geographical level, as well as the IRIS (infra-communal INSEE) and policy-related territories (Agglomerations, departments, regions ...).

Following this approach, MGDIS has been working on algorithms in order to determine the population potentially present in housing. The example above is used to indicate that an apartment of $50 \mathrm{~m}^{2}$, built before 1949 in Vannes (intramural), identified on the 3D map (cadastre and 3D representation), has a probability of $64 \%$ of using an electricity-based heating. 


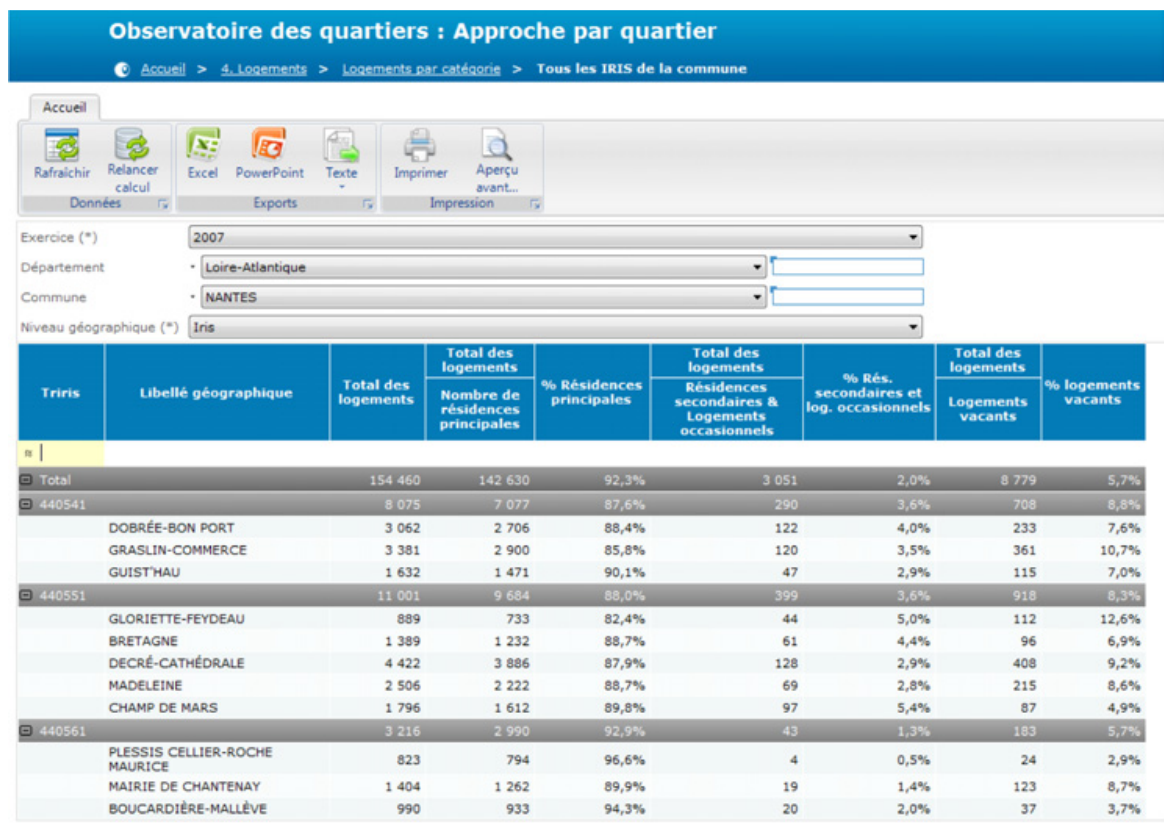

Figure 14. Screenshot of the MGDIS solution, enabling population localization from housing type.

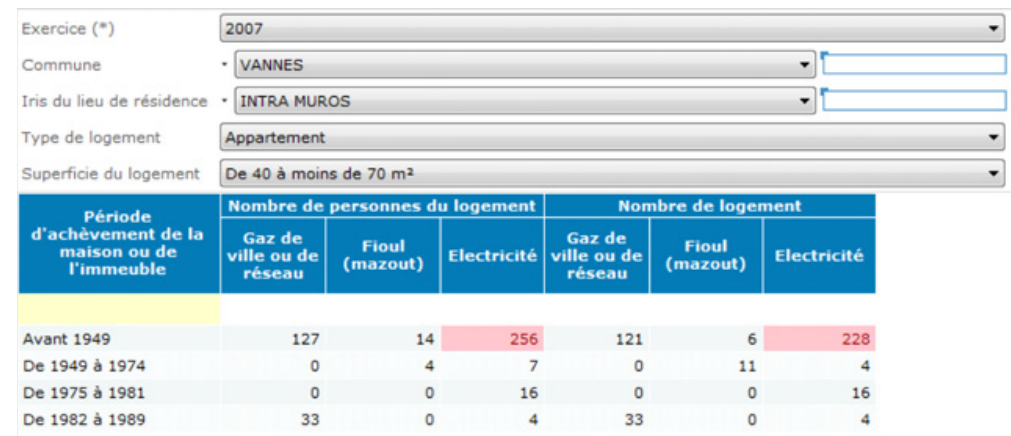

Figure 15. Screenshot of the MGDIS solution, enabling population localization from housing type.

\section{DISCUSSION}

At present, software solutions for communities help elected agents to define public policies by informing them about strengths and weaknesses in the territory. Through project EnVIE, fine-grained public data, together with a 3D representation of the territory and environmental constraints, allow precise actions to be taken, in order to reach goals defined in the community leading plan.

The EnVIE project aims at assisting institutional decision makers in framing and defining policies. Data collected by each partner will enable decision makers to conduct public policy for sustainable development, consistent with the needs of the population:

- Analysis of health risks: from environmental information (wind, sun exposure, building insulation) and demographic information (ages, isolation of the elderly...), elected members can identify 
risk areas and thus get closer to population during unexpected weather events (heat waves/cold weather).

- Energy efficiency: communities can identify buildings with low energy performance and help people equip themselves, with the goals of reducing the energy bill of the population and meeting the challenges defined in their sustainable development policy.

- Social mix: from this information, policymakers can foster communities in creating social mix of neighborhoods, thus maintaining a balance between all categories of people and all housing types.

\section{CONCLUSION}

The EnVIE project merges several techniques in order to produce critical indicators intended to be used into decision-making processes. A semi-automatic 3D acquisition technique produces a geometric 3DCM in CityGML format. This model is used to compute 3D metadata related to wind, lighting and thermic phenomenon. Data mining techniques are used to produce 2D metadata related socioeconomic data. The geometric model and the metadata are merged into a full 3DCM in order to build clear indicators. The main contribution of this project is to setup a full process from acquisition to visualization, and to mix metadata of various types.

All the processes have now to be automated through web services between projects partners. That is to say that each process will be seen as a web service, and therefore we will be able to produce any kind of metadata set from an unique portal, assuming the 3D data acquisition is done.

Where further works are concerned, the maintenance of the 3D data of the 3DCM is very challenging as the city is a dynamic entity in constant evolution. This challenge could be addressed with semantic model embedding the full history of the city allowing to represent all the changes that could occurs into a 3DCM.

This project is funded by Région Bretagne et Région Pays de Loire (Oséo).

\section{References}

[1] Bénitez, S., Denis, E., Baillard, C. (2010): "Automated Production of Occlusion-Free Rectified Façade Textures using Vehicle-based Imagery", Photogrammetric Computer Vision and Image Analysis, pp. 275.

[2] Meixner, M., Leberl, F. (2011): “3-Dimensional Building Details from Aerial Photography for Internet Maps”, Remote Sensing 2011, pp. 721-751.

[3] Corre, Y., Lostanlen, Y. (2009): "Three-Dimensional Urban EM Wave Propagation Model for Radio Network Planning and Optimization Over Large Areas", IEEE Transactions on Vehicular Technology, Vol. 58, No. 7, Sep. 2009, pp. 3112-3123.

[4] Caniot, G., Li, W., Dupont, G. (2011): "Validations and applications of a CFD tool dedicated to wind assessment in urban areas", 13th International Conference on Wind Engineering, 2011.

[5] Cherqui F., Groleau D., Wurtz E. et Allard F. (2005): "A step toward the global assessment of district projects: solar indicators and way to quantify them". Ninth International IBPSA Conference, Montrè̀al, Canada, Organizing Committee Building Simulation 2005, 15-18 aout 2005, p. 167-174.

[6] PACIBA: Progiciels d'Aide à la Conception Intégrée des Bâtiments, AAP ANR 2006 - Programme de Recherche sur l'Energie dans le Bâtiment (PREBAT).

[7] Miguet, F. \& Groleau, D. (2007): "Urban bioclimatic indicators for urban planers with the sofware tool SOLENE”. in: Luis, B., Manuel, P., Said, J., Ricardo, M. \& Rogério, A., (editors). Portugal 
Usage, Usability, and Utility of 3D City Models

SB07 Sustainable Construction, materials and practices: challenges of the industry for the new millenium, Lisbonne, 12-14. Amsterdam: IOS PRESS. p. 348-355.

[8] Király, G., Brolly, G. (2007): "Tree height estimation methods for terrestrial laser scanning in a forest reserve". International Archives of Photogrammetry, Remote Sensing and Spatial Information Sciences, Vol. 3 (Part 3/W52), pp. 211-215.

[9] Liberge, S., Soheilian, B., Chehata, N., Paparoditis, N. (2010): "Extraction of vertical posts in 3D laser point clouds acquired in dense urban areas by a mobile mapping system". International Archives of Photogrammetry, Remote Sensing and Spatial Information Sciences, Vol. 38(Part 3B), pp. 126-130. 